\title{
ERRATUM
}

\section{Sporidiobolus johnsonii and Sporidiobolus salmonicolor revisited}

\section{Elisabete Valério • Mário Gadanho •}

José Paulo Sampaio

Published online: 27 March 2008

(C) German Mycological Society and Springer-Verlag 2008

\section{Erratum to: Mycol Progress}

\section{DOI 10.1007/s11557-007-0547-8}

Due to an unfortunate turn of events, for which the authors assume full responsibility, the mating types of several strains were incorrect both in Table 1 and in Fig. 2. The table and figure are correctly displayed here and should be treated as definitive by the reader.

The online version of the original article can be found at: http://dx.doi. org/10.1007/s11557-007-0547-8.

E. Valério · M. Gadanho · J. P. Sampaio $(\bowtie)$

Centro de Recursos Microbiológicos,

Secção Autónoma de Biotecnologia,

Faculdade de Ciências e Tecnologia,

Universidade Nova de Lisboa,

2829-516 Caparica, Portugal, Spain

e-mail: jss@fct.unl.pt 
Table 1 List of strains used in this study and relevant information pertaining to them

\begin{tabular}{|c|c|c|c|c|}
\hline Strain & Species (phenotypic identification) & $\begin{array}{l}\text { Phylogenetic } \\
\text { group }\end{array}$ & Sexuality & Source \\
\hline CBS $5470^{\mathrm{T}}$ & S. johnsonii & JOHNS & Self-fertile & $\begin{array}{l}\text { Leaf of Rubus idaeus with dead pustule of } \\
\text { Phragmidium rubi-idaei, USA }\end{array}$ \\
\hline CBS 8241 & S. johnsonii & JOHNS & Self-fertile & Unknow \\
\hline CBS $1522^{\mathrm{T}}$ & Sp. Holsaticus & JOHNS & Asexual & Fodder yeast, Germany \\
\hline VKM Y-690 & S. salmonicolor & JOHNS & Asexual & Culture contaminant \\
\hline CBS 2641 & S. salmonicolor & JOHNS & Asexual & $\begin{array}{l}\text { Air, The Netherlands (deposited at CBS as } \\
\text { Sporobolomyces roseus Kluyver \& van Niel) }\end{array}$ \\
\hline CBS 2630 & S. salmonicolor & JOHNS & MT A2 & Air, The Netherlands \\
\hline CBS 2643 & S. salmonicolor & JOHNS & MT A2 & Isolated by $\mathrm{S}$. Windisch, Germany \\
\hline PYCC 4351 & S. salmonicolor & JOHNS & MT A2 & Tree leaf, Portugal \\
\hline CBS 4209 & S. salmonicolor & JOHNS & MT A2 & $\begin{array}{l}\text { Fruit body of Exidia sp., Japan (type strain } \\
\text { of Sporobolomyces coralliformis Tubaki) }\end{array}$ \\
\hline CBS $490^{\mathrm{T}}$ & S. salmonicolor & SALM & MT A1 & Culture contaminant \\
\hline CBS 483 & S. salmonicolor & SALM & MT A2 & $\begin{array}{l}\text { Rusted leaf of Citrus sp., France (type strain } \\
\text { of Sporobolomyces odorus Derx) }\end{array}$ \\
\hline CBS 487 & S. salmonicolor & SALM & MT A1 & $\begin{array}{l}\text { Air, Japan (type strain of Pseudomonilia } \\
\text { rubiduncula Okunuki) }\end{array}$ \\
\hline CBS 1039 & S. salmonicolor & SALM & MT A1 & Air in dairy, USA \\
\hline CBS 2634 & S. salmonicolor & SALM & Asexual & $\begin{array}{l}\text { Fragaria sp., Japan (deposited at CBS as } \\
\text { Sporidiobolus johnsonii Nyland) }\end{array}$ \\
\hline CBS 2873 & S. salmonicolor & SALM & MT A1 & $\begin{array}{l}\text { Extract of oak bark, France (type strain of } \\
\text { Sporobolomyces hispanicus } \\
\text { Peláez \& Ramírez) }\end{array}$ \\
\hline CBS 4029 & S. salmonicolor & SALM & MT A2 & $\begin{array}{l}\text { Soil, New Zealand (deposited at CBS as } \\
\text { Sporobolomyces odorus Derx) }\end{array}$ \\
\hline CBS 5937 & S. salmonicolor & SALM & MT A1 & $\begin{array}{l}\text { Carious dentine, South Africa (type strain of } \\
\text { Aessosporon salmonicolor van der Walt) }\end{array}$ \\
\hline CBS 6530 & S. salmonicolor & SALM & MT A1 & Bog, locality unknown \\
\hline CBS 6781 & S. salmonicolor & SALM & MT A1 & $\begin{array}{l}\text { Bituminous soil, Venezuela (type strain of } \\
\text { Sporidiobolus veronae Balloni, } \\
\text { Florenzano \& Materazzi) }\end{array}$ \\
\hline CBS 6832 & S. salmonicolor & SALM & MT A1 & $\begin{array}{l}\text { Cerebro-spinal fluid, India (type strain of } \\
\text { Sporobolomyces salmonicolor } \\
\text { (Fisher \& Brebeck) } \\
\text { Kluyver \& van Niel var. fischeri Misra \& Randhawa) }\end{array}$ \\
\hline CBS 7260 & S. salmonicolor & SALM & MT A1 & $\begin{array}{l}\text { USSR (type strain of Sporobolomyces philippovii } \\
\text { Krasil'nikov) }\end{array}$ \\
\hline PYCC 4558 & S. salmonicolor & SALM & MT A2 & Polluted river water, Oeiras, Portugal \\
\hline PYCC 5245 & S. salmonicolor & SALM & Asexual & Portugal \\
\hline RJB 948 & S. salmonicolor & SALM & MT A2 & $\begin{array}{l}\text { Isolated by R.J. Bandoni (deposited at CBS as } \\
\text { Sporobolomyces odorus Derx) }\end{array}$ \\
\hline RJB 950 & S. salmonicolor & SALM & MT A1 & $\begin{array}{l}\text { Isolated by R.J. Bandoni (deposited at CBS as } \\
\text { Sporobolomyces odorus Derx) }\end{array}$ \\
\hline RJB 8219 & S. salmonicolor & SALM & MT A1 & Isolated by R.J. Bandoni \\
\hline VKM Y-2291 & S. salmonicolor & SALM & MT A2 & Soil, USSR \\
\hline IHMT 2446/96 & S. salmonicolor & SALM & MT A2 & Skin lesion, Portugal \\
\hline ML 2241 & S. salmonicolor & SALM & MT A2 & Isolated by J.W. Fell \\
\hline ZP 392 & S. salmonicolor & SALM & MT A1 & Fruit body of Dacrymyces sp., Germany \\
\hline ZP 648 & S. salmonicolor & SALM & MT A2 & Leaf of Ligustrum sp., Monte de Caparica, Portugal \\
\hline
\end{tabular}


Fig. 2 Phylogenetic placement derived from maximum parsimony analysis (consensus tree of ten most parsimonious trees) of an expanded dataset of 31 representatives of Sporidiobolus johnsonii and $S$. salmonicolor based on complete ITS sequences.

Numbers on the branches are bootstrap values (1000 replicates; values below $50 \%$ are not shown). GenBank accession numbers of the sequences are indicated after strain numbers. The values of DNA-DNA reassociation experiments are indicated on the right side and the assimilation tests that are useful for the recognition of the two clades are indicated on the left side

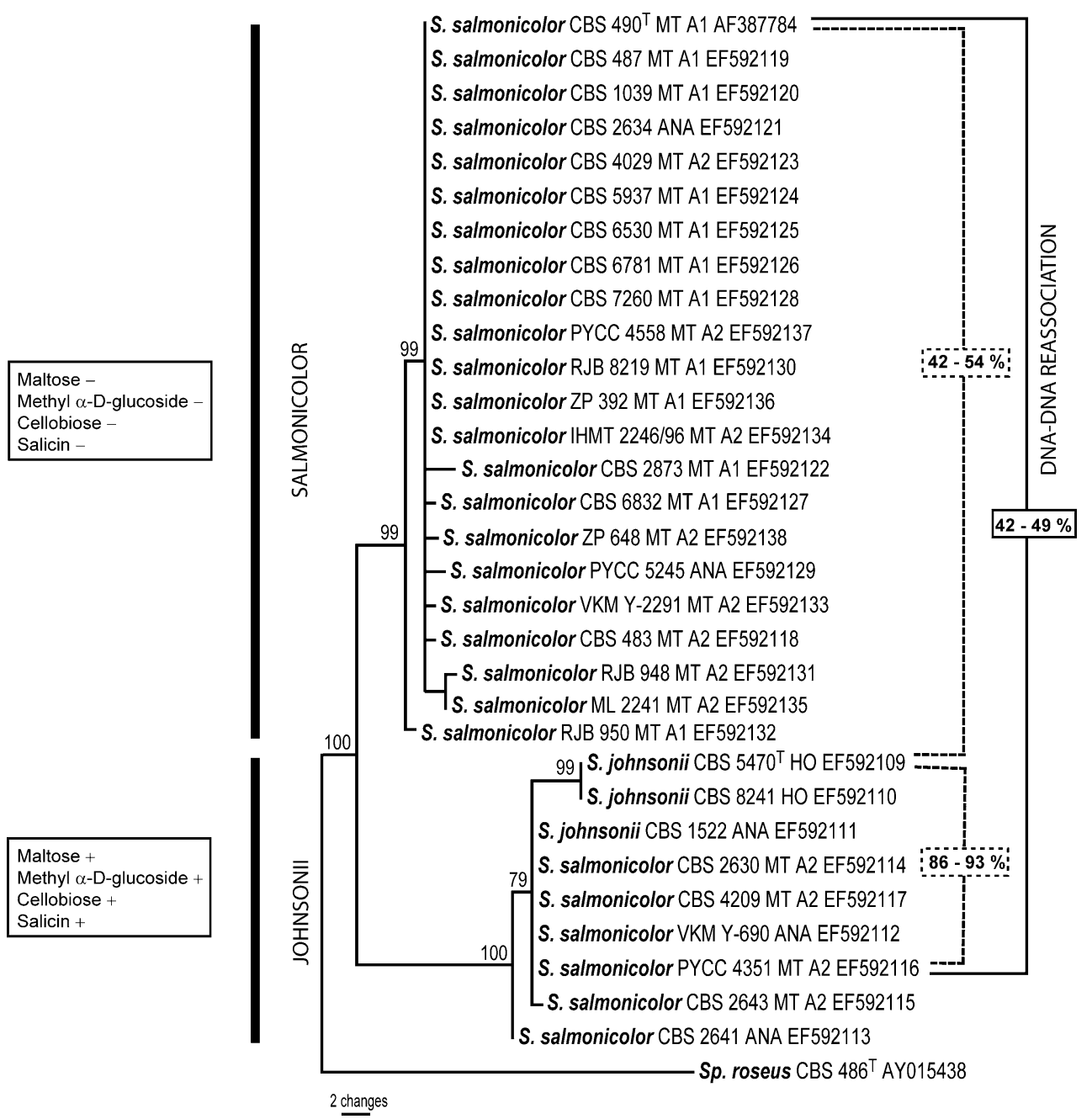

